\title{
CONTROL OF FILM GROWTH IN LITHIUM ION BATTERY PACKS VIA SWITCHES
}

\author{
Scott J. Moura* \\ Joel C. Forman \\ Jeffrey L. Stein Hosam K. Fathy \\ Automated Modeling/Control Optimization Laboratory \\ Department of Mechanical Engineering \\ University of Michigan \\ Ann Arbor, Michigan 48109 \\ Email: \{sjmoura,jcforman,stein,hfathy\}@umich.edu
}

\section{ABSTRACT}

Recent advances in lithium ion battery modeling suggest degradation may be reduced by permitting unequal but controlled charging of individual cells, delayed until immediately prior to discharge. Hence, this paper compares anode-side film formation for a standard equalization scheme versus unequal charging through switches - controlled by deterministic dynamic programming $(D D P)$ and DDP-inspired algorithms. A static model for film growth rate is derived from a first-principles battery model adopted from the electrochemical engineering literature. Coupled with this model, we consider two cells connected in parallel via relay switches. The key results we demonstrate are: (1) Enabling unequal charging and (2) delaying charging until immediately prior to discharge indeed reduces film buildup. (3) A near optimal control method can be implemented using heuristic rules designed from the DDP solution and convexity properties of film growth rate. Simulation indicates the heuristic rule achieves near optimal performance relative to the DDP solution, and over 50\% reduction in film growth compared to charging both cells equally.

\section{NOMENCLATURE}

\begin{tabular}{lll}
$F$ & Faraday's constant & {$[\mathrm{C} / \mathrm{mol}]$} \\
$i_{0}$ & Battery pack current & {$[\mathrm{A}]$} \\
$i_{0, s}$ & Exchange current density & {$\left[\mathrm{A} / \mathrm{m}^{2}\right]$} \\
$i_{1}, i_{2}$ & for side reaction & Cell current \\
$h_{\text {penalty }}$ & Quadratic penalty function & {$[\mathrm{A}]$} \\
\hline
\end{tabular}

$J \quad$ Cost functional $\quad\left[\mathrm{pm} / \mathrm{m}^{2}\right]$

$J_{1} \quad$ Intercalation current between $\quad\left[\mathrm{A} / \mathrm{m}^{3}\right]$

$\begin{array}{lll}J_{p} & \text { Terminal state penalty function } \quad\left[\mathrm{pm} / \mathrm{m}^{2}\right]\end{array}$

$J_{\text {tot }} \quad$ Total intercalation current $\quad\left[\mathrm{A} / \mathrm{m}^{3}\right]$

$J_{s} \quad$ Current density of side reaction $\quad\left[\mathrm{A} / \mathrm{m}^{3}\right]$

$M_{P} \quad \begin{aligned} & \text { Molecular weight of product } \\ & \text { from side reaction }\end{aligned} \quad[\mathrm{mol} / \mathrm{kg}]$

$Q \quad$ Battery cell charge capacity $\quad$ [A.h $]$

$q_{1}, q_{2} \quad$ Contactor switch position

$R \quad$ Universal gas constant $\quad[\mathrm{J} / \mathrm{K} / \mathrm{mol}]$

$R_{f i l m} \quad \begin{aligned} & \text { Total film resistance at } \\ & \text { electrode/electrolyte interface }\end{aligned} \quad\left[\Omega \cdot \mathrm{m}^{2}\right]$

$R_{\text {int }} \quad$ Battery cell internal resistance $\quad[\Omega]$

$R_{S E I} \quad$ Resistance of solid electrolyte $\quad\left[\Omega / \mathrm{m}^{2}\right]$

$\begin{array}{ll}U_{s, r e f} & \text { Equilibrium potential of } \\ \text { side reaction }\end{array}$

$V \quad$ Value function $\quad\left[\mathrm{pm} / \mathrm{m}^{2}\right]$

$\begin{array}{lll}v & \text { Battery pack voltage }\end{array}$

$x \quad$ Spatial coordinate $\quad[\mathrm{m} / \mathrm{m}]$

$z \quad$ Battery cell state of charge $\quad[\mathrm{C} / \mathrm{C}]$

$\Delta \phi \quad$ Local potential difference between $\quad[\mathrm{V}]$

$\delta_{\text {film }}$ Resistive film thickness $\quad\left[\mathrm{pm} / \mathrm{m}^{2}\right]$

$\eta_{s} \quad$ Over potential driving side reaction $\quad$ [V]

$\kappa_{P} \quad$ Conductivity of electrolyte $\quad[1 / \mathrm{m} / \Omega]$

$\rho_{P} \quad$ Density of product from side reaction $\left[\mathrm{kg} / \mathrm{m}^{2}\right]$

\footnotetext{
*Address all correspondence to this author.
} 


\section{INTRODUCTION}

This paper examines health management algorithms for reducing film buildup in battery packs, consisting of two modules connected in parallel. Battery health improvement represents one of the key opportunities for catalyzing the integration of plugin electric vehicles with the electricity infrastructure (vehicle-togrid, or V2G) [1]. Moreover, improving battery lifetime may substantially reduce the required size (manufacturers might currently oversize batteries to compensate for capacity fade) and replacement period of high-capacity battery packs. This is particularly important for ensuring the financial feasibility of plug-in electric vehicles and, more generally, energy storage solutions for the electric grid. Therefore we seek to design battery pack management algorithms that control degradation in some optimal sense. In this paper we consider lithium ion chemistries, which have been identified as a promising battery technology for achieving high energy and power densities, among other benefits [2]. Managing degradation is particularly challenging because the associated mechanisms, including resistive film growth at the anode, are typically simulated using computationally intensive electrochemistry-based models. Moreover, it is currently impractical to directly access and control the mechanisms causing degradation inside the cell. To reconcile these issues, we propose a nearly optimal feedback control algorithm for reducing degradation that utilizes a simple degradation model with existing relays in high energy capacity battery packs. At the cell-tocell level, researchers have proposed charge equalization circuits that protect cells connected in series strings from over-charging or over-discharging due to capacity imbalances [3,4]. This paper proposes an additional battery health management algorithm at the cell module level. Namely, we consider the potential advantages of allowing unequal charge values across modules connected in parallel. Hence, this paper extends research on battery health management by adding four important and original contributions. First, we utilize a high fidelity physics-based electrochemical model of film growth to generate a reduced model for control. Second, we propose to utilize existing relay switches in battery packs, typically designed for thermal runaway, for active control of film growth. Third, we pose an optimal control problem that seeks to minimize total battery pack film growth through appropriate relay switching sequences. Fourth, we demonstrate that a nearly optimal control policy can be implemented as a set of heuristic rules, designed from the optimal control results and convexity properties of film growth rate. Literature on lithium ion batteries contains several models for capacity and power fade. However, this paper uses an electrochemical-based model that considers a specific and important source of degradation anode-side film growth. Physics-based mathematical models of health degradation in Li-ion cells have been studied extensively in the electrochemical literature. A popular model for capturing the lithium diffusion dynamics and intercalation phenomena was developed by Doyle, Fuller, and Newman in [5,6]. This model is particularly appealing because it is generalizable to different cathode materials and physical parameters, for anodes com-

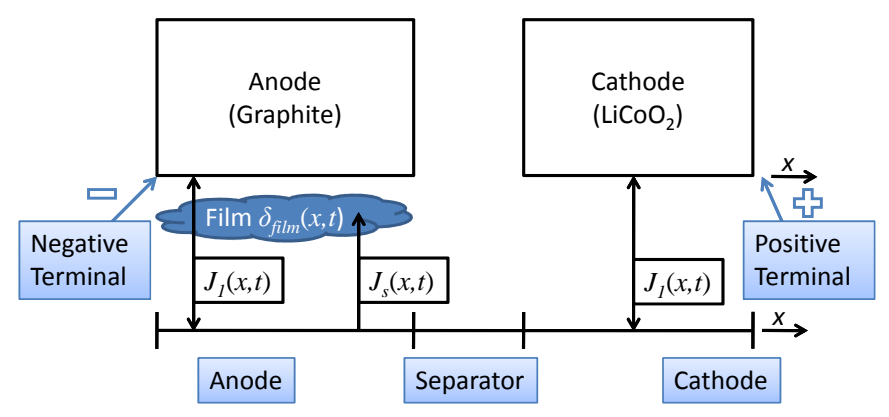

Figure 1. STRUCTURE OF THE ELECTROCHEMICAL LITHIUM-ION BATTERY CELL MODEL.

posed of ethylene carbonate. However, the initial versions did not consider the mechanisms causing capacity loss and power fade. Consequently, Ramadass et al. [7] extended this model by hypothesizing an irreversible solvent reduction reaction at the anode-side electrode/electrolyte interface that generates a resistive film by consuming cyclable lithium. Since this process is considered one of the chief contributors to capacity fade and power loss, this paper uses the model presented in [7] to study battery health management.

Although there have been few publications on controlling battery battery health, the concept of modeling battery degradation in terms of charge capacity fade and increased internal resistance spawned a body of research known as state-ofhealth ( $\mathrm{SOH}$ ) estimation. Research on $\mathrm{SOH}$ estimation generally uses empirical equivalent circuit battery cell models to estimate charge capacity and internal resistance, using a variety of algorithms, such as batch data reconciliation, moving-horizon parameter estimation [8], recursive least squares [9], and extended Kalman filtering [10-12]. Recently, Smith, Rahn, and Wang [13] used a standard Kalman filter to estimate the internal spatialtemporal states of an electrochemical model derived from [5, 6]; however this work does not estimate $\mathrm{SOH}$-related parameters.

The aim of this paper is to extend dynamic systems battery health research into the arena of lithium-ion film growth control. As such, the remainder of this paper is organized as follows: Section 2 reviews an electrochemical model for film growth developed by Ramadass, et al. [7], the reduced model utilized in this paper, and a simple battery pack design. Section 3 formulates a deterministic dynamic programming (DDP) problem for minimizing total film growth in the battery pack under consideration. Section 4 analyzes the DDP results and interprets the solution via the convexity properties of film growth rate. This analysis motivates the design of a suboptimal heuristic feedback control law. Section 5 characterizes the performance of the heuristic algorithm vis-a-vis the DDP solution and standard charge equalization scheme. Finally, Section 6 summarizes the paper's main conclusions. 


\section{MODEL DEVELOPMENT}

\subsection{Electrochemical Capacity Fade Mechanics}

In this paper, a function mapping cell state of charge (SOC) and current to film growth rate is extracted from a first-principles electrochemical Li-ion battery cell model developed in [7]. This model simulates phenomena such as lithium ion diffusion and intercalation to determine the potential and concentration gradients in the solid and solution sections of the anode, cathode, and separator. A schematic of the cell model is provided in Fig. 1, where Ramadass et al. argue that a resistive film builds up on the anode electrode/electrolyte interface [7]. The exact chemical side reaction depends on the chemistry of the electrode and electrolyte. Equations (1)-(6), developed by Ramadass et al. argue that a very simple and general method for modeling capacity loss is to assume an irreversible solvent reduction reaction of the following form

$$
S+L i^{+}+e^{-} \rightarrow P
$$

where $S$ denotes the solvent species and $P$ is the product.

As a result of this irreversible side reaction, the products form a film at the electrode/electrolyte interface. This film has a time and spatially varying thickness $\delta_{\text {film }}(x, t)$, which, combined with the solid electrolyte interphase (SEI) resistance $R_{S E I}$, models the total resistance at the electrode/electrolyte interface as follows

$$
R_{f i l m}(x, t)=R_{S E I}+\frac{\delta_{f i l m}(x, t)}{\kappa_{P}}
$$

where $\kappa_{P}$, denotes the conductivity of the film, $x$ is the spatial coordinate, and $t$ is time. The state equation corresponding to the growth of film thickness, due to the unwanted solvent reduction described in Eq. (1), is given by

$$
\frac{\partial \delta_{\text {film }}(x, t)}{\partial t}=-\frac{M_{P}}{a_{n} \rho_{P} F} J_{s}(x, t)
$$

In Eq. (3), $M_{P}, a_{n}, \rho_{P}$, and $F$ represent, the product's molecular weight, specific surface area, mass density, and Faraday's constant, respectively. The term $J_{s}$ denotes the local volumetric current density for the side reaction, which is governed by Butler-Volmer kinetics. If we assume the solvent reduction reaction is irreversible and the variation of Li-ion concentration in the solution is small, then we may approximate $J_{s}$ by the following Tafel equation [14].

$$
J_{s}(x, t)=-i_{0, s} a_{n} e^{\left(\frac{-0.5 F}{R T} \eta_{s}(x, t)\right)}
$$

In Eq. (4), $i_{0, s}, R$, and $T$ respectively denote the exchange current density for the side reaction, universal gas constant, and cell temperature. The term $\eta_{s}$ represents the side reaction overpotential, which drives the solvent reduction reaction in Eq. (1). This variable is expressed by the following equation, based on Kirchoff's voltage law.

$$
\eta_{s}(x, t)=\Delta \phi(x, t)-U_{s, r e f}-\frac{J_{t o t}(x, t)}{a_{n}} R_{f i l m}(x, t)
$$

The variable $\Delta \phi$ represents the difference in potentials between the solid and solution. The symbol $U_{s, \text { ref }}$ denotes the equilibrium potential of the solvent reduction reaction, which we assume to be constant. The total intercalation current $J_{t o t}$ models the flow of charge exchanged with the anode-side solution. Specifically, the total intercalation current $J_{t o t}$ is given by the sum of current between the solid and solution $\left(J_{1}\right)$, and the solvent reduction reaction and solution $\left(J_{s}\right)$, that is

$$
J_{t o t}=J_{1}+J_{s}
$$

Equations (2)-(6) encompass the film growth subsystem of the Li-ion battery cell model, adopted from [7]. This subsystem connects to the remainder of the battery model through the total intercalation current $J_{t o t}$ and potential difference $\Delta \phi$. Since these variables vary with respect to space (across the electrodes and separator) and time, they are determined by solving coupled partial differential equations and algebraic constraints representing the concentration and potential distributions in the solid and solution of the anode, cathode, and separator (see $[5,6]$ for details). Although this model accurately predicts electrochemical phenomena such as diffusion dynamics and film growth, its complexity makes control design for health management difficult. Therefore, the present research seeks to use the high fidelity model to generate simpler models for the purposes of control design. To acquire insight on the relationship between battery cell SOC, current, and film growth rate, consider a fresh cell, that is $\delta_{f i l m}(x, 0)=0$. Also assume all the intercalation currents, overpotentials, and concentration profiles are constant with respect to space and correspond to zero applied current. Under these assumptions, we simulate the electrochemical battery cell model to compute a static relationship mapping cell SOC and applied current to the spatially averaged film growth rate $\dot{\bar{\delta}}_{\text {film }}$, shown in Fig. 2. The map indicates that film growth rate increases with cell SOC. The film growth rate also increases as the discharge current becomes increasingly negative, i.e. for increasing charge current. Finally, film grows when zero current is applied, indicating that aging occurs even when the cells are not in use. A key question we revisit after obtaining the optimal control solution is what insight can be extracted from this map to design controllers that reduce film formation in battery packs?

\subsection{Battery Pack Model}

Switched capacitor circuits $[3,4]$ are typically applied to equalize individual SOC levels for cells connected in series. In 


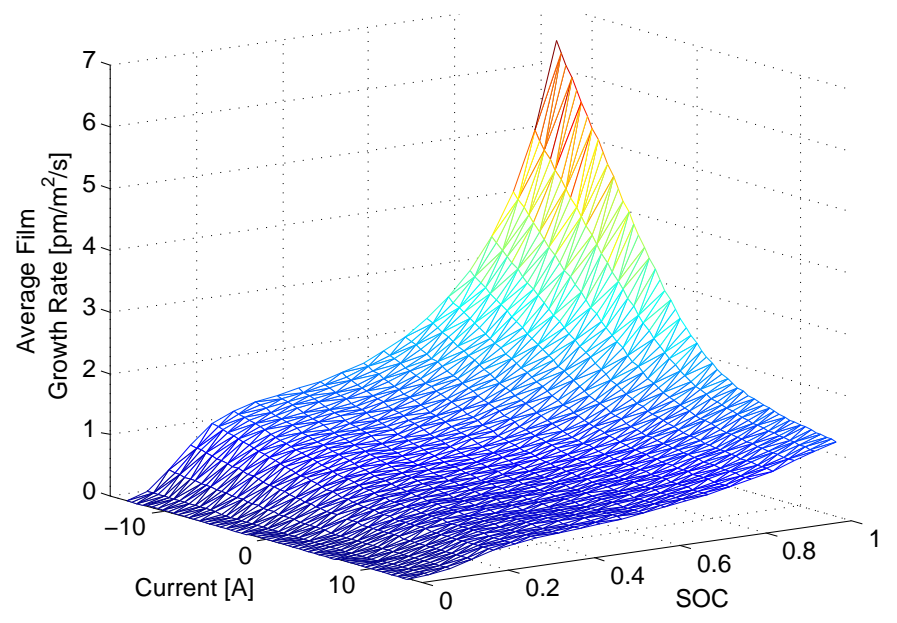

Figure 2. STATIC APPROXIMATION OF FILM GROWTH RATE VS. CELL CURRENT AND SOC FOR FRESH CELL (i.e. $\delta_{\text {film }}=0$ ). POSITIVE CURRENT CORRESPONDS TO DISCHARGE.

this paper, we examine the potential advantages of allowing unequal charge levels for battery modules connected in parallel. A simple method to independently control module charge levels uses switches in protection circuits [15] (e.g. solid state relays or contactors). These devices are primarily designed to disconnect the battery in case of imminent catastrophic behavior, such as thermal runaway [16]. When multiple modules are arranged in parallel, individual solid state relays can be connected in series with each parallel branch. These relays may serve as one potential opportunity for individually controlling battery module SOC, and will be the topology we consider henceforth.

Consider a battery pack architecture consisting of two modules connected in parallel through two switches, where each module contains one cell for simplicity (Fig. 3). The goal is to determine the optimal switching strategy that minimizes the total film growth of both cells, given an exogenous current trajectory $i_{0}$. Due to the computational complexity of the distributed parameter electrochemical cell model described in Section 2.1, and the curse of dimensionality imposed by dynamic programming [17], we require a simplified model for control design. As such, we utilize an equivalent circuit model $[11,18]$, written in discrete time, with a ten second time step $(\Delta T=10 \mathrm{sec})$. This equivalent circuit model consists of an open circuit voltage source $O C V$ in series with an internal resistor $R_{\text {int }}$. Open circuit voltage and internal resistance are nonlinear functions of SOC, that is $O C V\left(z_{i}\right)$ and $R_{\text {int }}\left(z_{i}\right)$ where $i=1,2$. The state variables $z_{1}$ and $z_{2}$ represent the SOC of battery cells 1 and 2 respectively. The dynamic equations for each cell are based on integrating current $i_{1}, i_{2}$ to obtain charge, and then dividing by the total charge

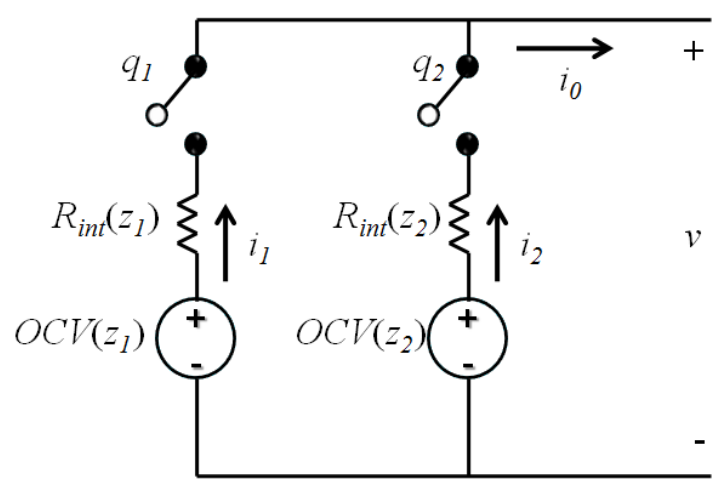

Figure 3. CIRCUIT DIAGRAM OF BATTERY PACK.

capacity of the cell $Q$.

$$
\begin{aligned}
& z_{1, k+1}=z_{1, k}-\frac{i_{1, k}}{Q} \Delta T \\
& z_{2, k+1}=z_{2, k}-\frac{i_{2, k}}{Q} \Delta T
\end{aligned}
$$

The currents $i_{1}, i_{2}$ are determined by the configuration of the switches and exogenous current demand on the battery pack $i_{0}$. The currents are given by Kirchoff's current law, where the switching signals $q_{1}$ and $q_{2}$ equal zero and one when the corresponding switch is respectively open or closed:

$$
\begin{aligned}
i_{1, k} & =q_{1, k}\left(1-q_{2, k}\right) i_{0, k} \\
& +\frac{O C V\left(z_{1, k}\right)-O C V\left(z_{2, k}\right)+i_{0, k} R_{\text {int }}\left(z_{1, k}\right)}{2 R_{\text {int }}\left(z_{1, k}\right)} q_{1, k} q_{2, k} \\
i_{2, k} & =\left(1-q_{1, k}\right) q_{2, k} i_{0, k} \\
& +\frac{O C V\left(z_{2, k}\right)-O C V\left(z_{1, k}\right)+i_{0, k} R_{\text {int }}\left(z_{2, k}\right)}{2 R_{\text {int }}\left(z_{2, k}\right)} q_{1, k} q_{2, k}
\end{aligned}
$$

The first terms on the right-hand side of (9), (10) model one cell connected at a time. The second terms model both cells connected at the same time. Note that the battery does not charge (i.e. experiences zero current) when both $q_{1}$ and $q_{2}$ equal zero. The parameters $O C V$ and $R_{\text {int }}$ for the equivalent circuit model are identified from the full electrochemical model described in Section 2.1. Similar to the film growth rate map in Fig. 2, these parameters assume constant potential and concentration distributions for a given SOC value. The open circuit voltage is given by the potential in the solid portion of the cathode, at the positive terminal, for zero applied current, shown in (11). The internal resistance is computed via Ohm's law in (12). Each cell has a charge capacity of $Q=1.8 \mathrm{Ah}$.

$$
\begin{aligned}
O C V(z) & =\Delta \phi_{\text {cathode }}(x=L) \\
R_{\text {int }}(z) & =\frac{\left(\Delta \phi_{\text {cathode }}(x=L)-O C V(z)\right)}{i_{\text {app }}}
\end{aligned}
$$


The symbol $L$ denotes the length of the cathode, $i_{a p p}$ is the current applied to the battery cell, and each electrode is initialized at concentration levels corresponding to a cell SOC level of $z$. Using these measurements, we fit $O C V$ and $R_{\text {int }}$ with the following polynomial regression models:

$$
\begin{aligned}
\text { OCV }(z)= & 9.62 z^{5}-27.7 z^{4}+30.4 z^{3} \\
& -15.4 z^{2}+3.90 z+3.37 \\
R_{\text {int }}(z)= & -0.319 z^{5}+0.989 z^{4}-1.15 z^{3} \\
& +0.645 z^{2}-0.188 z+0.114
\end{aligned}
$$

\section{OPTIMAL CONTROL PROBLEM FORMULATION}

The control objective is to determine the optimal switching sequence that minimizes the total resistive film growth in the battery pack described in Section 2, given a current trajectory, $i_{0}$, known a priori. We formulate this as a finite horizon constrained optimal control problem

$$
\begin{aligned}
\min _{q_{1}, q_{2}} J= & \sum_{k=1}^{N}\left[\dot{\bar{\delta}}_{f i l m}\left(z_{1, k}, i_{1, k}\right)+\dot{\bar{\delta}}_{f i l m}\left(z_{2, k}, i_{2, k}\right)+g_{z}\left(z_{k}\right)\right] \\
& +\alpha_{N}\left\|z_{N}-0.95\right\|_{2}^{2}
\end{aligned}
$$

subject to

$$
\begin{aligned}
z_{k+1} & =f\left(z_{k}, i_{k}\right) \\
i_{k} & =h\left(q_{k}, i_{0, k}\right) \\
z_{1} & =z_{i c}
\end{aligned}
$$

where

$$
\begin{aligned}
\left(q_{1}, q_{2}\right) \in & \{0,1\} \times\{0,1\} \\
g_{z}\left(z_{k}\right)= & \alpha_{z}\left[\sum_{i=1,2} \max \left\{0.05-z_{i, k}, 0, z_{i, k}-0.98\right\}\right]^{2}+ \\
& \alpha_{v}\left[\sum_{i=1,2} \max \left\{2.0-v_{i, k}, 0, v_{i, k}-4.3\right\}\right]^{2} \\
z_{k}= & {\left[z_{1, k} z_{2, k}\right]^{T} } \\
i_{k}= & {\left[i_{1, k} i_{2, k}\right]^{T} }
\end{aligned}
$$

where the function $\dot{\bar{\delta}}_{f i l m}$ maps SOC and current to average film growth rate according to the relationship depicted in Fig. 2. The function $g_{z}\left(z_{k}\right)$ denotes soft constraints that limit cell SOC and cell voltage to protect against over-charging and overdischarging. However, for the simulation described in this paper, these constraints never become active due to the modest $1 \mathrm{C}$ charging rate employed. A terminal constraint with weighting $\alpha_{N}$ is provided to ensure the battery pack charges to the SOC corresponding to the desired final voltage. The function $f\left(z_{k}, i_{k}\right)$ represents the dynamic equation in (7)-(8). The function $h\left(q_{k}, i_{0, k}\right)$ maps the switch position and battery pack current to cell current in (9)-(10). Finally, we impose a fixed initial condition $z_{i c}$.

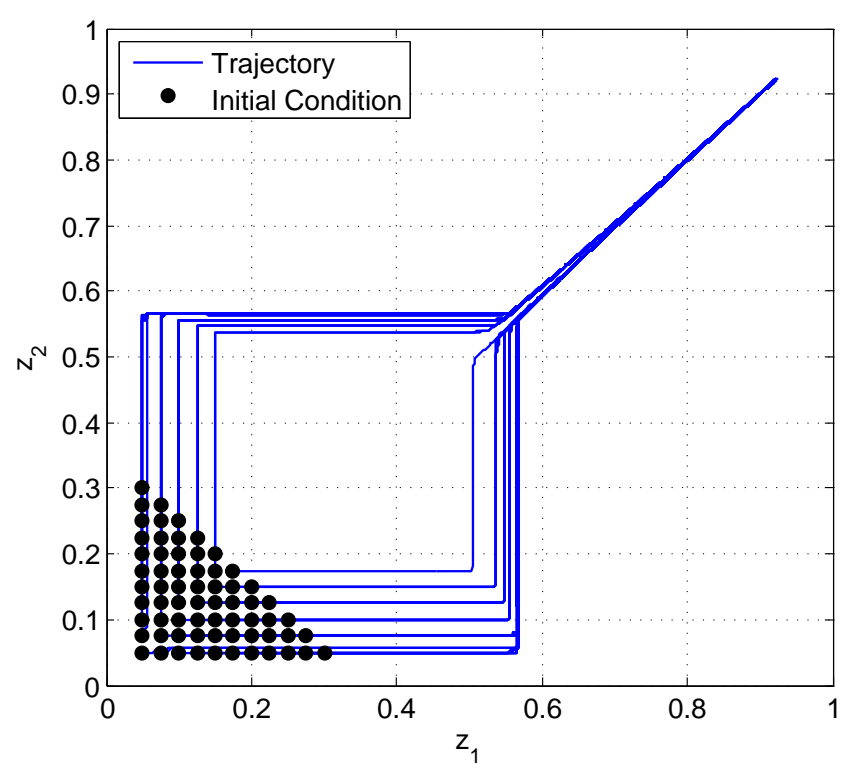

Figure 4. OPTIMAL TRAJECTORIES FOR VARIOUS INITIAL CONDITIONS, GIVEN A 1C BATTERY PACK CHARGE RATE.

To solve the optimization problem in (15)-(22), we reexpress the equations as a dynamic programming problem by defining a value function as follows [17]: Let $V_{k}\left(z_{k}\right)$ represent the minimum total film growth from discrete time $k$ to the end of the time horizon, given that the cell SOC in the present time step $k$ is given by the vector $z_{k}$. Then the optimization problem can be written as the following recursive optimality equation and boundary condition.

$$
\begin{aligned}
V_{k}\left(z_{k}\right) & =\min _{q_{1}, q_{2}}\left\{\begin{array}{c}
\dot{\bar{\delta}}_{f i l m}\left(z_{1, k}, i_{1, k}\right)+\dot{\bar{\delta}}_{f i l m}\left(z_{2, k}, i_{2, k}\right) \\
+g_{z}\left(z_{k}\right)+V_{k+1}\left(z_{k+1}\right)
\end{array}\right\} \\
V_{N}\left(z_{N-1}\right) & =\min _{q_{1}, q_{2}}\left\{\alpha_{N}\left\|z_{N}-0.95\right\|_{2}^{2}\right\}
\end{aligned}
$$

The above dynamic programming problem is solved via a full enumeration algorithm. That is, we compute a family of optimal trajectories for a set of fixed initial conditions. This approach enables us to analyze an ensemble of trajectories to gain insight on how DDP minimizes total film growth.

\section{SOLUTION ANALYSIS}

\subsection{Analysis of Optimal Trajectories}

To acquire insight on the optimal switching sequence for minimizing resistive film growth, we consider a constant $1 \mathrm{C}$ (1.8 A) charge rate applied to the battery pack. Note that while the battery pack experiences a constant current charge rate, the individual cells will have time varying charge rates. Figure 4 demonstrates the optimal trajectories for a set of initial battery cell SOC conditions. Time responses for an initial SOC of 0.1 for each cell 

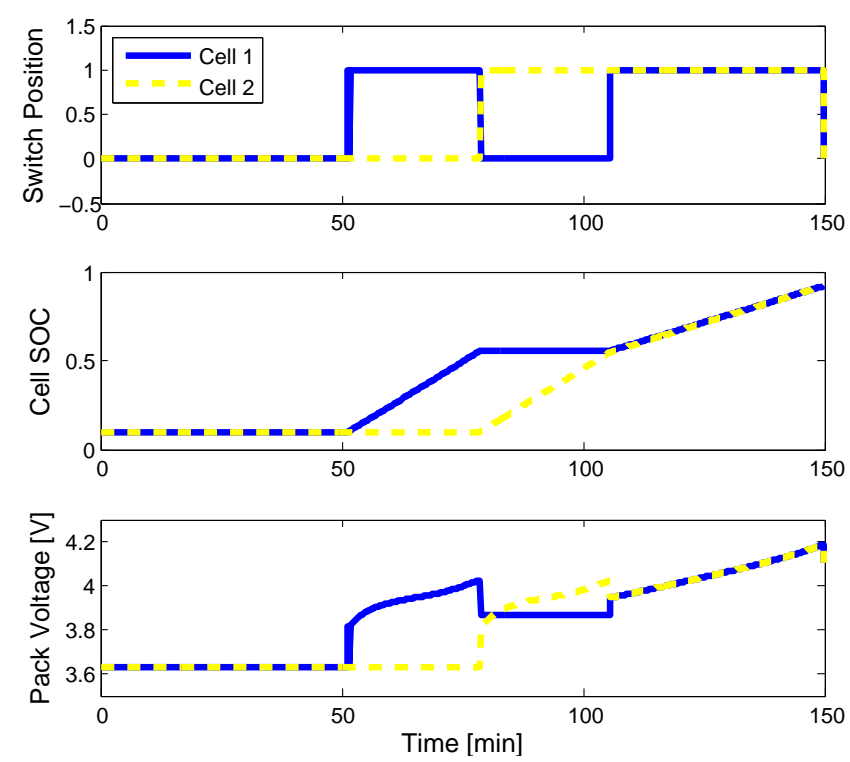

Figure 5. TIME RESPONSES FOR OPTIMAL CHARGING PATTERN IDENTIFIED BY DDP, GIVEN A 1C BATTERY PACK CHARGE RATE.

are provided in Fig. 5. These figures indicate that the optimal switching sequence follows a consistent pattern:

1. Leave the battery pack uncharged for as long as possible.

2. Charge the cell with greater initial SOC.

3. Charge the cell with less SOC until both cells approximately equalize.

4. Charge both cells together, at approximately equal SOC values, until the final state is reached.

The key question is why does DDP identify the aforementioned pattern as the optimal switching sequence for minimizing film growth?

\subsection{The Energy Storage-Film Growth Tradeoff}

First, let us focus our attention on the result that film growth is minimized by leaving the battery pack uncharged for as long as possible. In other words, film growth is minimized if battery packs are charged only immediately before use. The reason for this result can be seen by observing that the film growth rate increases with SOC in Fig. 2. Therefore, maintaining each cell in a low SOC reduces the overall film buildup. However, this requires a priori knowledge of when the battery pack will be used. Moreover, if the battery is discharged sooner than expected, only a fraction of the total energy capacity is available for use. This suggests a fundamental tradeoff between electric energy storage and reducing anode-side film growth.

\subsection{Convexity Analysis of Film Growth Rate}

Next, let us focus on the switching pattern exhibited by the optimal solution when charging does occur. Consider the film growth rate for varying SOC and zero current input, as portrayed

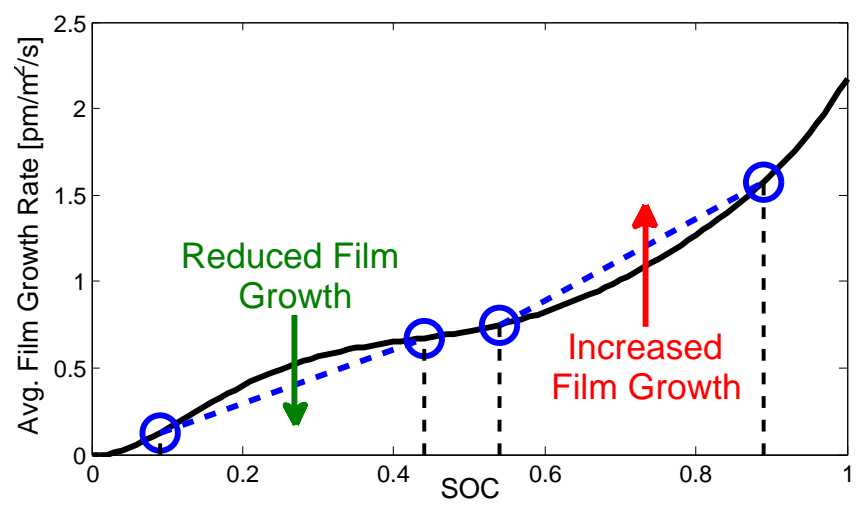

Figure 6. CONVEXITY ANALYSIS OF SPATIALLY-AVERAGED FILM GROWTH RATE FOR ZERO APPLIED CURRENT.

in Fig. 6. For small SOC values, $\dot{\bar{\delta}}_{\text {film }}$ is concave. Along this portion of the curve, the total film growth rate for two cells at different SOC values is less than the total film growth rate for two cells at the same SOC value. However, for large SOC values $\dot{\bar{\delta}}_{f i l m}$ is convex. This implies that the total film growth rate for two cells at different SOC values is greater than the total film growth rate for two cells at equal SOC values. If one assumes the solution is infinitely greedy, these observations for reducing film growth can be applied as follows:

1. In the concave region of $\dot{\bar{\delta}}_{f i l m}$, drive the individual SOC values apart.

2. In the convex region of $\dot{\bar{\delta}}_{\text {film }}$, equalize the individual SOC values.

These results indicate that a reduction in total film growth can be achieved by allowing individual modules to have unequal SOC values. Additionally, the optimal policy follows a consistent pattern that may be approximated by a heuristic feedback control law, which leaves the battery discharged for the maximum allowable time.

\subsection{DDP-inspired Heuristic Control}

Inspired by these results, and the convexity analysis presented in Section 4.2, we examine a heuristic control scheme for minimizing film growth, depicted in Fig. 7. The advantage of a heuristic control scheme over the optimal trajectories computed by DDP is that the former can be implemented in a feedback loop. Additionally, one expects the heuristic scheme to achieve nearly optimal performance, due to the consistent pattern exhibited by the DDP solutions. The process of converting optimal trajectories into an explicit feedback map has been studied extensively in model predictive control theory [19]. These concepts are potentially applicable here, but a simpler less formal approach is used in this initial study. Note that the switching pattern defined by the heuristic rule should not be initiated until the last possible opportunity. In this example, each cell has a 1.8 A-h charge capacity and thus the total pack charge capacity is 3.6 


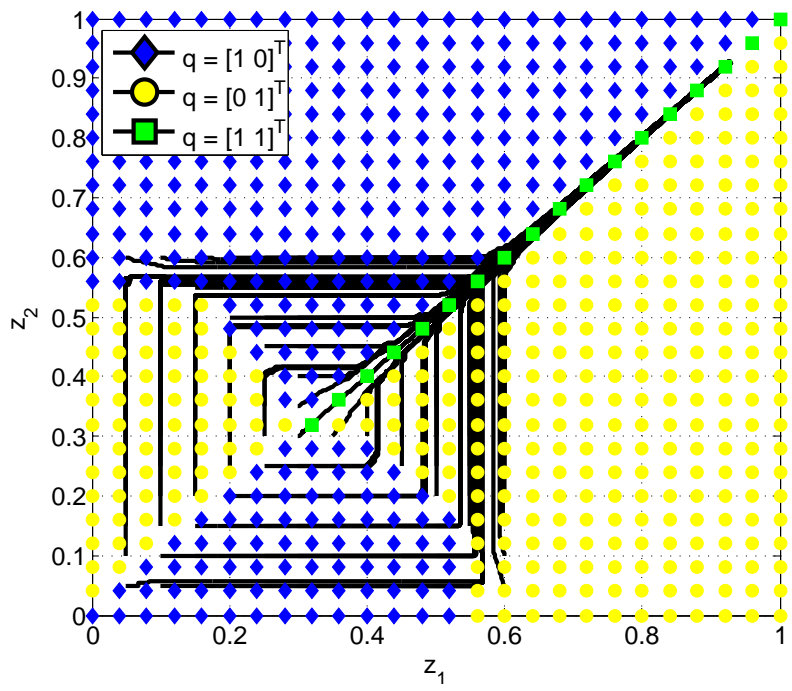

Figure 7. DDP-INSPIRED HEURISTIC RULE FOR CHARGING, WITH OPTIMAL STATE TRAJECTORIES SUPERIMPOSED.

A-h. Therefore charging both cells from $0.1 \mathrm{SOC}$ to $0.95 \mathrm{SOC}$ at a $1 \mathrm{C}(1.8 \mathrm{~A})$ rate requires about 100 minutes. As a result, we initiate the heuristic charging scheme 100 minutes prior to the final time.

The design of the heuristic control law follows two steps: First, we simulate the optimal trajectories from a family of initial conditions, such as shown in Fig. 4. Second, we identify regions of the state-space corresponding to a certain switch configuration. For regions in which the optimal state trajectories do not enter, we select a switch configuration that steers the state toward an optimal trajectory. This step is required, because for the $1 \mathrm{C}$ charge rate input studied here, feasible trajectories do not cover the entire state-space. The final result of this procedure is depicted in Fig. 7, where several optimal state trajectories are superimposed on the proposed heuristic rule.

\section{SIMULATION RESULTS}

To evaluate the performance of the proposed heuristic controller, we compare it to the optimal DDP-based and standard equalization schemes (i.e. both switches closed during charging). We perform this study by simulating the closed loop battery pack degradation control system for a $1 \mathrm{C}(1.8 \mathrm{~A})$ constant current charge rate cycle. The initial cell SOC values are 0.1 each. In practice, the standard charge method is to apply constant current to every cell in the pack until the voltage reaches a maximum value, then the voltage is held constant at this maximum value until the applied current reaches some minimal level. This is known as a constant current, constant voltage (CCCV) charge cycle [7]. Here, we only investigate the potential improvements incurred during the period when the cells charge up to a maximum voltage limit, corresponding to $0.95 \mathrm{SOC}$ in our simulations.

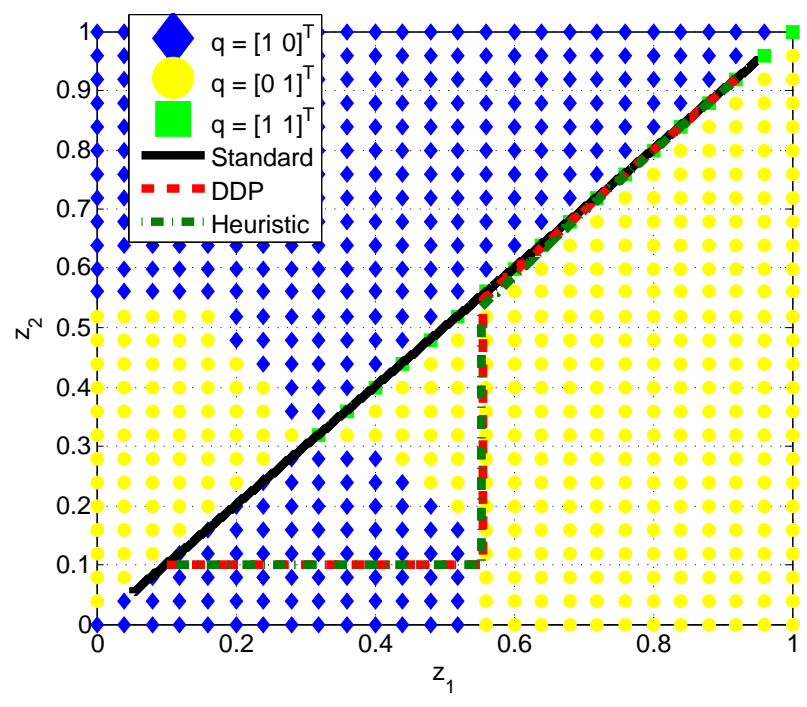

Figure 8. SOC TRAJECTORIES FOR EACH CONTROL SCHEME, SUPERIMPOSED ON THE HEURISTIC CONTROL MAP.

\subsection{Charge Cycle Simulation}

The cell SOC trajectories for each control scheme are provided in Fig. 8, superimposed on the heuristic rule. Observe that the standard charging scheme maintains each cell at equal SOC values as the battery pack charges. In contrast, the trajectories corresponding to DDP and the heuristic rule follow trajectories similar to Fig. 4 and 7. Namely, both methods charge one cell at a time in the concave region of $\dot{\bar{\delta}}_{\text {film }}$, and then apply charge equalization in the convex region of $\dot{\bar{\delta}}_{f i l m}$. Also observe that trajectories for DDP and the heuristic controller match closely, indicating that the proposed heuristic controller closely approximates the optimal solution for the trajectory shown here. Time responses for the cell SOC, current, and battery pack voltage are provided in Fig. 9. Here we see that the heuristic rule is initiated 50 minutes into the simulation, allowing 100 minutes of charging time. All three subplots further demonstrate how closely the heuristic controller and DDP solution match, with respect to time. Since the standard method initiates charging immediately, the cells remain idle at $0.95 \mathrm{SOC}$ once charging is complete. This is important because film builds up at a faster rate for high SOC relative to low SOC. The impact of this property can be seen in the third subplot. The second subplot demonstrates each cell's voltage, which increases only when that particular cell is charging. Note that all schemes maintain the cell voltage within the safety range of $2.0 \mathrm{~V}$ to $4.3 \mathrm{~V}$.

\subsection{Discussion of Performance Results}

A comparison of the performance for each control scheme is provided in Table 1. For the $1 \mathrm{C}$ rate charge cycle studied in this paper, the heuristic controller produces an additional $7 \mathrm{p} \Omega / \mathrm{m}^{2}$ of resistive film buildup over the DDP solution. Hence, the heuristic scheme exhibits nearly identical performance to the optimal 

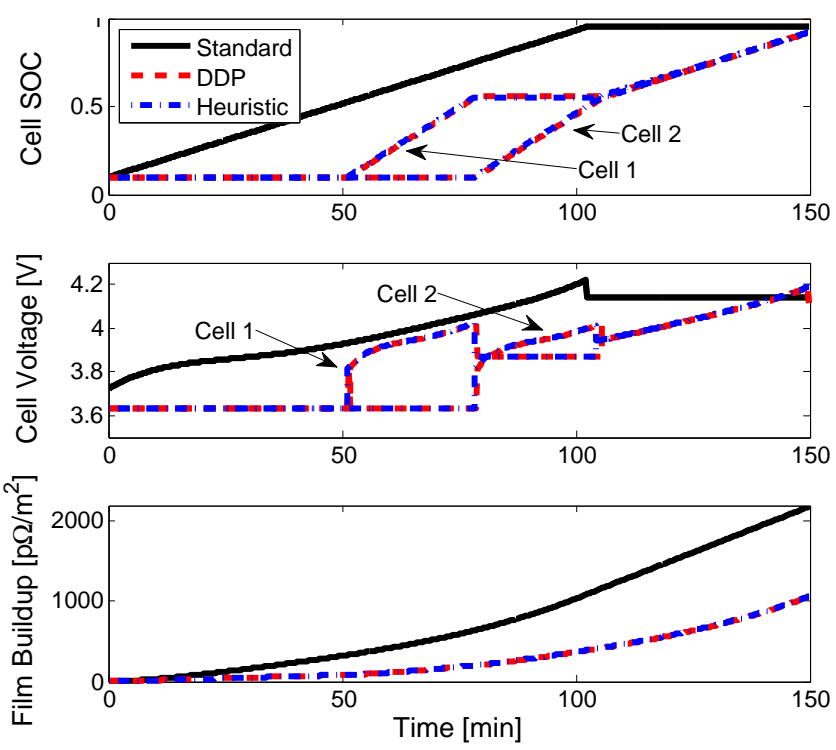

Figure 9. TIME RESPONSES FOR EACH CONTROL SCHEME.

control design. Both DDP and the heuristic controller reduce film buildup by over $50 \%$, for this charge cycle. It is important to note that the reduction in film buildup is a function of the particular charge cycle and time horizon. That is, cycles that remain within the concave region of $\dot{\bar{\delta}}_{f i l m}$ will experience greater improvement, because the switched scheme proposed in this paper has the greatest advantage in this domain. Moreover, the bulk of film reduction occurs due to delaying the charging process to the end of the time horizon. For the example studied here, $48 \%$ of film buildup reduction is due to delayed charging and $2 \%$ due to the unequal charging pattern. More importantly, the conclusion that battery SOC should remain low to minimize film buildup introduces an interesting tradeoff between electrochemical energy storage and battery health. Finally, we note that although the switched schemes reduce total film buildup, they sacrifice some power efficiency. In particular, the DDP and heuristic scheme reduce power efficiency by over $1 \%$. This result occurs due to increased $i^{2} R$ losses that occur when applying greater current values through each cell's internal resistance. Hence, there exists a tradeoff between long term battery degradation and short

Table 1. CONTROLLER PERFORMANCE COMPARISON.

\begin{tabular}{llll}
\hline \hline $\begin{array}{l}\text { Control } \\
\text { Scheme }\end{array}$ & $\begin{array}{l}\text { Resistance of } \\
\text { Total Film } \\
\text { Buildup }\end{array}$ & $\begin{array}{l}\text { Reduction } \\
\text { in Film } \\
\text { Buildup }\end{array}$ & $\begin{array}{l}\text { Average } \\
\text { Power } \\
\text { Efficiency }\end{array}$ \\
\hline \hline Standard & $2182 \mathrm{p} \Omega / \mathrm{m}^{2}$ & $0 \%$ & $98.0 \%$ \\
DDP & $1058 \mathrm{p} \Omega / \mathrm{m}^{2}$ & $51.5 \%$ & $96.8 \%$ \\
Heuristic & $1065 \mathrm{p} \Omega / \mathrm{m}^{2}$ & $51.2 \%$ & $96.8 \%$ \\
\hline \hline
\end{tabular}

term power efficiency.

Although the degradation reduction techniques demonstrated in this paper are admittedly preliminary, the analysis motivates several health management ideas currently under investigation. For example, this work assumes cell current can only be controlled through relay switches, thus constraining current to take on values in a discrete set. However, we are currently considering more advanced circuitry that enables a continuous split of current between parallel cells, thus allowing greater freedom in navigating the film growth map. Secondly, the distributed parameter nature of the electrochemical cell model enables the use of theory developed for boundary control of PDE's [20]. Finally, model reduction techniques (e.g. [21]) are currently under consideration for properly balancing model fidelity with amenability to control design.

\section{CONCLUSIONS}

This research investigates battery health management in lithium ion battery packs using relay switches for modules connected in parallel. To facilitate control design and analysis, we consider an electrochemical battery cell model with irreversible solvent reduction reaction dynamics at the anode, developed by Ramadass et. al. [7]. From this high fidelity model, we approximate film growth rate as a static map that functionally depends on cell SOC and applied current. From this map, we formulate an optimal control problem to minimize total battery pack film growth for a constant current charge trajectory. Inspired by the optimal trajectories, and the convexity properties of the film growth map, we design a heuristic rule base that produces nearly optimal performance.

The key results demonstrated by this work is that health degradation due to film growth can be reduced by: (1) Allow battery modules connected in parallel to attain unequal SOC values. (2) Delay charging until immediately before discharging. Indeed, the optimal solution approximately separates SOC in the concave region and equalizes SOC in the convex region of film growth rate at the end of the time horizon. Individual control of module SOC is achieved via relay switches typically used for safety precautions. Within each module, individual cell SOC may be equalized via traditional switched capacitor circuits $[3,4]$ to protect against over-charging or over-discharging. Simulation results indicate this approach may reduce film growth by over $50 \%$.

\section{ACKNOWLEDGMENT}

This research is financially supported by the National Science Foundation Graduate Research Fellowship and Emerging Frontiers in Research and Innovation (EFRI) - Resilient and Sustainable Infrastructures (RESIN) programs (Grant No. \#0835995).

\section{REFERENCES}

[1] Sovacool, B. K., and Hirsh, R. F., 2009. "Beyond batteries: An examination of the benefits and barriers to plug-in hy- 
brid electric vehicles (PHEVs) and a vehicle-to-grid (V2G) transition”. Energy Policy, 37(3), 3, pp. 1095-1103.

[2] Alamgir, M., and Sastry, A. M., 2008. "Efficient batteries for transportation applications". SAE Paper 2008-21-0017.

[3] Kimball, J. W., Kuhn, B. T., and Krein, P. T., 2007. "Increased performance of battery packs by active equalization”. 2007 IEEE Vehicle Power and Propulsion Conference, Sept. 9-12 2007.

[4] Baughman, A., and Ferdowsi, M., 2008. "Double-tiered switched-capacitor battery charge equalization technique". IEEE Trans. on Industrial Electronics, 55(6), pp. 2277-85.

[5] Doyle, M., Fuller, T., and Newman, J., 1993. "Modeling of galvanostatic charge and discharge of the lithium/polymer/insertion cell". Journal of the Electrochemical Society, 140(6), pp. 1526 - 33.

[6] Fuller, T., Doyle, M., and Newman, J., 1994. "Simulation and optimization of the dual lithium ion insertion cell". Journal of the Electrochemical Society, 141(1), pp. 1- 10.

[7] Ramadass, P., Haran, B., Gomadam, P., White, R., and Popov, B., 2004. "Development of first principles capacity fade model for Li-ion cells". Journal of the Electrochemical Society, 151(2), pp. $196-203$.

[8] Gatzke, E., Stamps, A., Holland, C., and White, R., 2005. "Analysis of capacity fade in a lithium ion battery". Journal of Power Sources, 150, 10/04, pp. 229-39.

[9] Verbrugge, M., and Tate, E., 2004. "Adaptive state of charge algorithm for nickel metal hydride batteries including hysteresis phenomena". Journal of Power Sources, 126(1-2), pp. 236-249.

[10] Plett, G. L., 2004. "Extended Kalman filtering for battery management systems of LiPB-based HEV battery packs. Part 1. Background". Journal of Power Sources, 134(2), 08/12, pp. 252-61.

[11] Plett, G., 2004. "Extended Kalman filtering for battery management systems of LiPB-based HEV battery packs. Part 2. Modeling and identification". Journal of Power Sources, 134(2), pp. $262-76$.

[12] Plett, G. L., 2004. "Extended Kalman filtering for battery management systems of LiPB-based HEV battery packs. Part 3. State and parameter estimation". Journal of Power Sources, 134(2), 08/12, pp. 277-92.

[13] Smith, K., Rahn, C., and Wang, C.-Y., 2008. "Modelbased electrochemical estimation of lithium-ion batteries". 2008 IEEE International Conference on Control Applications (CCA) part of the IEEE Multi-Conference on Systems and Control, pp. $714-19$.

[14] Newman, J., 1991. Electrochemical Systems, 2nd ed. Prentice Hall.

[15] Balakrishnan, P., Ramesh, R., and Prem Kumar, T., 2006. "Safety mechanisms in lithium-ion batteries". Journal of Power Sources, 155(2), pp. 401-414.

[16] Kim, G.-H., Pesaran, A., and Spotnitz, R., 2007. "A threedimensional thermal abuse model for lithium-ion cells". Journal of Power Sources, 170(2), pp. 476 - 89.
[17] Denardo, E., 2003. Dynamic Programming: Models and Applications. Courier Dover Publications.

[18] Moura, S. J., Fathy, H. K., Callaway, D. S., and Stein, J. L., 2008. "A stochastic optimal control approach for power management in plug-in hybrid electric vehicles". ASME Dynamic Systems and Control Conference, Oct. 2008.

[19] Bemporad, A., Borrelli, F., and Morari, M., 2002. "Model predictive control based on linear programming - the explicit solution". IEEE Transactions on Automatic Control, 47(12), pp. 1974 - 1985.

[20] Krstic, M., and Smyshlyaev, A., 2008. Boundary Control of PDEs: A Course on Backstepping Design. SIAM.

[21] Cai, L., and White, R. E., 2009. "Reduction of model order based on proper orthogonal decomposition for lithium-ion battery simulations". Journal of the Electrochemical Society, 156(3), pp. A154 - A161. 